\title{
Fluorescent Nanodiamond-Nanogels for Nanoscale Sensing and Photodynamic Applications
}

Yingke Wu, ${ }^{\dagger}$ Md Noor A Alam, 'Priyadharshini Balasubramanian, Pia Winterwerber, Anna Ermakova, Michael Müller, Manfred Wagner, Fedor Jelezko*, Marco Raabe*, and Tanja Weil*

Y. Wu, M. N. A. Alam, M. Raabe, P. Winterwerber, Dr. A. Ermakova, M. Müller, Dr. M. Wagner, Prof. Dr. T. Weil

Max Planck Institute for Polymer Research, Ackermannweg 10, 55128 Mainz, Germany

*E-mail: weil@mpip-mainz.mpg.de, raabe@mpip-mainz.mpg.de

Y. Wu, M. N. A. Alam, M. Raabe, Prof. Dr. T. Weil

Institute of Inorganic Chemistry I, Ulm University, Albert-Einstein-Allee 11, $89081 \mathrm{Ulm}$, Germany

Priyadharshini Balasubramanian, Prof. Dr. F. Jelezko

Institute for Quantum Optics and IQST, Ulm University, Albert-Einstein-Allee 11, $89081 \mathrm{Ulm}$, Germany

*E-mail: fedor.jelezko@uni-ulm.de

Keywords: Nanodiamonds, nanogels, adsorption-crosslinking, nanoscale sensing, photodynamic applications

\begin{abstract}
Fluorescent nanodiamonds (NDs) are carbon-based nanoparticles with various outstanding magneto-optical properties. After preparation, NDs have a variety of different surface groups that determine their physicochemical properties. For biological applications, surface modifications are crucial to impart a new interphase for controlled interactions with biomolecules or cells. Herein, a straight-forward synthesis concept denoted "adsorptioncrosslinking" is applied for the efficient modification of NDs, which sequentially combines fast non-covalent adsorption based on electrostatic interactions and subsequent covalent crosslinking. As a result, a very thin and uniform nanogel coating surrounding the NDs is obtained, which imparts reactive groups as well as high colloidal stability. The impact of the reaction time, monomer concentration, molecular weight, and structure of the cross-linker on the resulting nanogel shell, the availability of reactive chemical surface functions and the quantum sensing properties of the coated NDs has been assessed and optimized. Post-modification of the
\end{abstract}


nanogel-coated NDs was achieved with phototoxic ruthenium complexes yielding ND-based probes suitable for photodynamic applications. The adsorption-crosslinking ND functionalization reported herein provides new avenues towards functional probes and traceable nanocarriers for high resolution bioimaging, nanoscale sensing and photodynamic applications.

\section{Introduction}

Among many carbon-based nanoscale materials, fluorescent nanodiamonds (NDs) have emerged for diverse applications in nanomedicine and bioimaging, because of their unique magneto-optical properties as well as their high biocompatibility. ${ }^{[1]}$ The optically active atom defects in the lattice of NDs, such as the nitrogen vacancy (NV) center, provide stable fluorescence without photobleaching or photoblinking. ${ }^{[2]}$ Due to their stable fluorescence, NDs with NV centers have been applied widely in bioimaging ${ }^{[3]}$ as well as real-time reporters for drug delivery. ${ }^{[4-6]}$ In addition, the emission wavelength of NDs is size-independent but tuneable from the visible to the near infrared region based on the color center (e.g., Si, Ge, etc.). ${ }^{[7]}$ Furthermore, NDs containing negatively charged $\mathrm{NV}\left(\mathrm{NV}^{-}\right)$centers can serve as single-spin sensors to detect critical physical parameters in a biological microenvironment, such as temperature, ${ }^{[8]}$ magnetic fields, ${ }^{[9]}$ electron spins, ${ }^{[10]}$ and mechanical strain. ${ }^{[11]}$

However, for most of these applications, an appropriate surface functionalization of the NDs is essential, because the colloidal stability of unmodified nanosized NDs in physiological buffer systems is extremely poor due to aggregation. ${ }^{[12]}$ Moreover, the shell provides further reactive groups to attach the desired functionalities, such as drug molecules, dyes, cell or tissue targeting groups or various proteins like antibodies. ${ }^{[13]}$ The surface coating shields the inner ND surface and creates a new interface, which is particularly attractive for in vitro or in vivo applications, i.e. to prevent foreign body interactions, ${ }^{[14]}$ to increase their circulation times ${ }^{[12]}$ or their accumulation at the target site ${ }^{[15]}$. In the past, various different ND surface coating materials and strategies have been reported. The covalent attachment of functionalities such as a silica 
shell, ${ }^{[16]}$ hyperbranched polyglycerols (HPG), ${ }^{[17]}$ poly(L-DOPA), ${ }^{[5]}$ and antibodies ${ }^{[18]}$ has been explored as well as non-covalent adsorption of biomolecules or polymers like polyethyleneimine,${ }^{[19]}$ insulin, ${ }^{[20]}$ and albumin-based copolymers. ${ }^{[4]}$ Nevertheless, uncontrolled aggregation as well as precipitation still represents a challenge and each reported functionalization strategy has its benefits but also inherent limitations. Covalent conjugation approaches are typically challenged by the low number of functionalities intrinsically present at the ND surface. ${ }^{[21]}$ Therefore, often only few surface groups could be attached and batch-tobatch variations of the NDs can cause reproducibility problems. ND coating by physical adsorption is a straight forward procedure, which is less influenced by changes of the ND surface groups. However, ligand loss often occurs in biological media thus limiting the stability of the nanoparticle i.e. during cell studies. ${ }^{[22]}$ We propose that the combination of both covalent and non-covalent coating approaches offers efficient functionalization and stabilization of NDs. Herein, we report a straight forward procedure based on the non-covalent adsorption and covalent crosslinking method that imparts a nanogel shell at the ND surface. First, the adsorption of multifunctional and positively charged ligands precoats the NDs based on electrostatic interactions followed by a cross-linking step to afford a stable and soft nanogel shell. Hyperbranched polyethyleneimine (PEI) is a highly branched, cationic polymer with multiple primary amino groups that allows cellular uptake by endocytosis, which has been widely used in biomedical applications, such as cell transfection ${ }^{[23]}$ and gene therapy ${ }^{[24]}$. In addition, to avoid aggregation of NDs during the adsorption of PEI, polyvinylpyrrolidone (PVP) has been applied as a commonly used stabilizer ${ }^{[25]}$ with proven biocompatibility as demonstrated in biomedical applications such as in tissue engineering. ${ }^{[26]}$ PEI has been crosslinked with poly(ethylene glycol) to generate the soft and homogeneous ND-nanogel shell providing high colloidal stability, reactive primary amino groups for post modifications and low cellular toxicity. We have accomplished a ND-nanogel platform for the design of ND-based photosensitizers for photodynamic applications (PDT). Photodynamic therapy is widely applied 
in skin cancer and here, light energy is converted locally at the tumor site into reactive oxygen species that affect cancer cell viability. ${ }^{[27]}$ In the future, real-time monitoring of the local changes in ion concentration of certain protein markers i.e. upon drug treatment could give new insights into cellular processes during therapy. In this way, a traceable ND-based photodynamic agent combining sensing, imaging and drug delivery offers great prospects for nanomedicine.

\section{Results and Discussion}

The adsorption and crosslinking procedure to generate a nanogel shell around NDs is depicted in Figure 1A. First, the NDs are mixed with hyperbranched polyethyleneimine (PEI, MW: $25 \mathrm{kDa}$ ) in the presence of polyvinylpyrrolidone (PVP, MW: $10 \mathrm{kDa}$ ) in Milli-Q water. At slightly alkaline (pH7.4) in the presence of phosphate-buffered saline (PBS), the 4-arm PEGNHS ester was applied to cross-link the surface adsorbed PEI for about 90 min. Purification was accomplished by several washing steps to remove unreacted precursors and smaller nanogels without the embedded ND in order to obtain the pure ND-nanogels (ND-NGs), as listed in Table S1.

The ND and ND-NGs were characterized with regards to their distribution, shape, and morphology by transmission electron microscopy (TEM; Figure 1B and 1C). As shown in Figure 1B, TEM images revealed that bare NDs were prone to significant aggregation and displayed a heterogeneous distribution whereas non-aggregating, homogeneous single particles could be observed for ND-NGs (Figure 1C). High-resolution transmission electron microscopy (HRTEM) imaging showed a uniform ring of $2 \mathrm{~nm}$ surrounding the NDs indicating the presence of a dry nanogel coating (Figure S1). Because the shell was low in contrast and measured under dry condition by TEM, we further assessed the core-shell structure of the ND-NGs by liquid mode atomic force microscopy (AFM; Figure 1D and 1E), in which the nanogel could swell compared to the dry TEM conditions. In Figure 1D, the topographic image of ND-NGs showed a good distribution with no obvious aggregation. The height-sensor images recorded by AFM 
were converted into deformation images using NanoScope Analysis 1.8 software to visualize the nanogel coating. ${ }^{[28]}$ Apart from the height profile images, further nanomechanical properties were simultaneously recorded. In particular, the deformation of the sample caused by the probe was analyzed to receive in-depth information on the structure of the coated NDs. As the ND core is much harder than the nanogel shell, the deformation of the nanogel shell could be detected with greater intensity under the same stress conditions. The deformation image revealed clearly that all NDs were uniformly surrounded by a soft shell (Figure 1D, right). Furthermore, the deformation image was studied at higher magnification (Figure 1E) and approximately $10 \mathrm{~nm}$ thickness of the nanogel was determined in liquid, which is sufficiently thin for nanoscale sensing applications. 

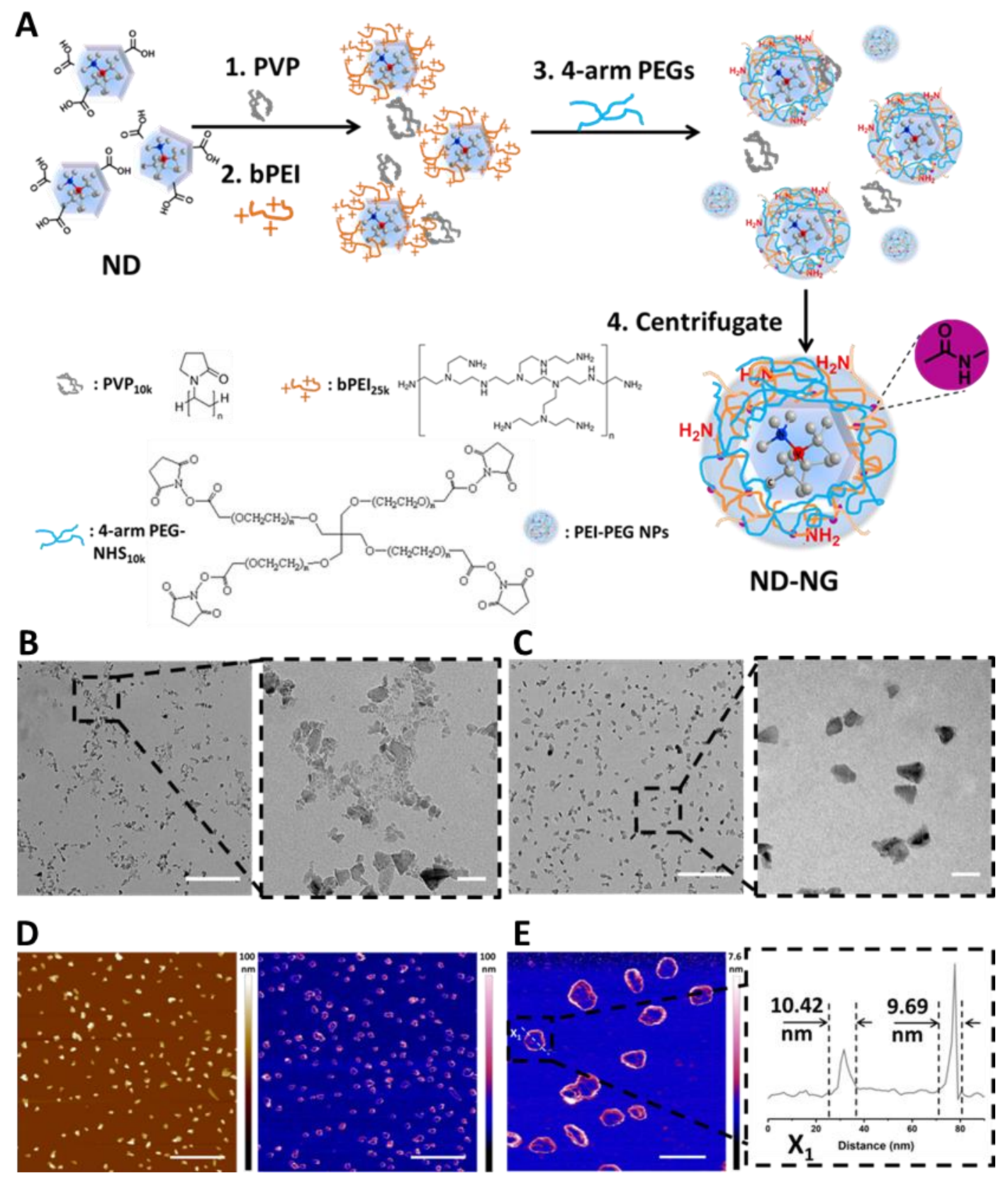

Figure 1. (A) Schematic illustration of the preparation of ND-NGs. (B) TEM images of NDs (left: scale bar $=500 \mathrm{~nm}$; right: scale bar $=50 \mathrm{~nm}$ ). (C) TEM images of ND-NGs (left: scale bar $=500 \mathrm{~nm}$; right: scale bar $=50 \mathrm{~nm}$ ). (D) AFM images of ND-NGs at liquid state (left: height sensor; right: deformation; scale bar $=500 \mathrm{~nm}$ ). (E) AFM images of ND-NGs (left: scale bar=100 nm; right: deformation distance curve of ND-NGs).

The ND-NGs were characterized by dynamic light scattering (DLS) to measure the shell thickness of dispersed ND-NGs in aqueous solution. The hydrodynamic diameters in Milli-Q water of NDs and ND-NGs was $36.2 \pm 2.4 \mathrm{~nm}$ and $57.0 \pm 1.2 \mathrm{~nm}$, respectively, resulting in a shell thickness of $10.4 \pm 3.6 \mathrm{~nm}$ of the ND-NGs (Figure $\mathbf{2 A}$ ). The ND-NGs were also characterized by multi-angle light scattering, which is more sensitive to detect aggregation. No 
aggregate formation was observed and a shell thickness of about $9 \mathrm{~nm}$ (Figure S2) was determined, which are in agreement with the results from the AFM measurements $(10 \mathrm{~nm})$. Variation of the reaction conditions on the hydrodynamic diameters of the ND-NGs was investigated by DLS. As shown in Figure S3, their sizes ND-NGs increased with the reaction time from $45.7 \pm 5.6 \mathrm{~nm}$ to $52.2 \pm 4.0 \mathrm{~nm}$. However, the increments slowed down gradually and stabilized after 90 min suggesting that the reaction reached saturation or completion. When the concentration of the cross-linker was increased from 0.125 to $8 \mathrm{mg} \mathrm{mL}^{-1}$, ND-NGs of 57.0 $\pm 1.2 \mathrm{~nm}$ were obtained with $2 \mathrm{mg} \mathrm{mL}^{-1}$ but the sizes subsequently increased to $74.9 \pm 9.9 \mathrm{~nm}$ for $8 \mathrm{mg} \mathrm{mL}^{-1}$ cross-linker. In addition, the PEI concentrations were also varied from 0.01 to $10 \mathrm{mg} \mathrm{mL}^{-1}$ to assess the influence on the resulting colloidal stability (Figure 2B). Aggregation was detected at a concentration of ND-NGs of $0.01 \mathrm{mg} \mathrm{mL}^{-1}$, most likely because the low amount of PEI around single ND was insufficient to impart sufficient colloidal stability. At 1 $\mathrm{mg} \mathrm{mL} \mathrm{mL}^{-1}$, ND-NGs with dimensions of about $50.2 \pm 6.0 \mathrm{~nm}$ were detected indicating that a sufficient amount of PEI covered the surface of the NDs thus forming a thin shell. Further increase in PEI to $10 \mathrm{mg} \mathrm{mL}^{-1}$ resulted in a significantly thicker shell $(160.9 \pm 22.6 \mathrm{~nm})$. The influence of the molecular weight (MW) of PEI and a regularly branched polyamidoamine (PAMAM) dendrimer with a globular sphere-like structure (PAMAM-G3) as well as the buffer, which was used to prepare ND-NGs, were investigated (Figure S4). NDs aggregated and precipitated when PEIs with lower MW were used (PEI 600 Da and PEI 2000 Da). Alternatives, such as the positive charged dendrimer PAMAM-G3, did not improve colloidal stability and precipitation was likewise observed, implying that only the positive charged branched polymer with appropriate MW (PEI 25,000 Da) and molecular structure allowed the preparation of stable ND-NGs. The ionic strengths of the buffer affected the preparation of ND-NGs as well (Figure 2A, Table S2), yielding ND-NGs with various sizes in Milli-Q water (83.3 $\pm 11.0 \mathrm{~nm})$, PBS $(57.0 \pm 1.2 \mathrm{~nm})$, and HEPES $(59.6 \pm 6.7 \mathrm{~nm})$. 
A

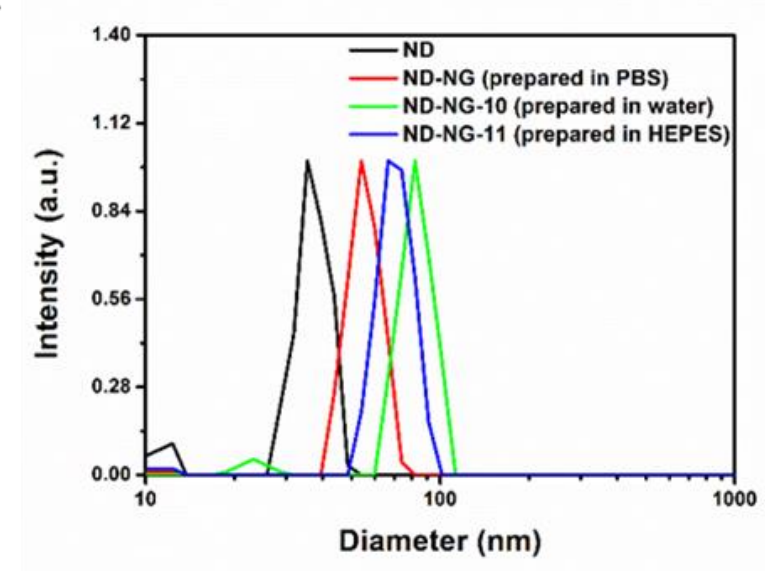

C

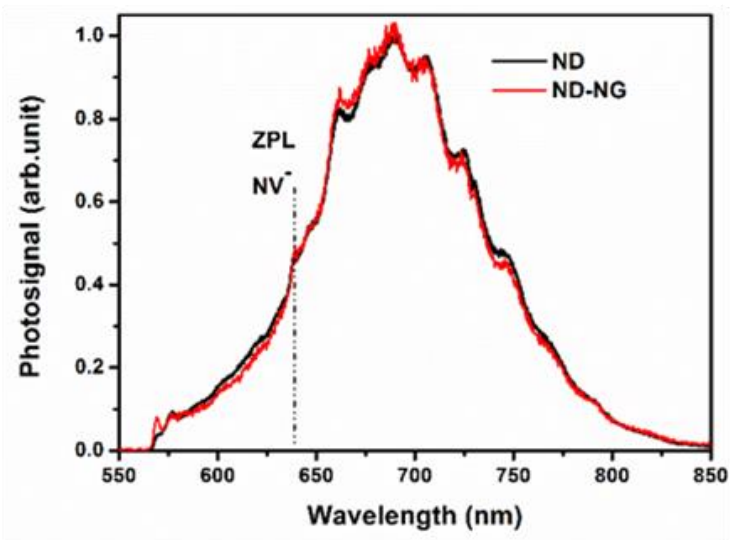

$\mathbf{E}$

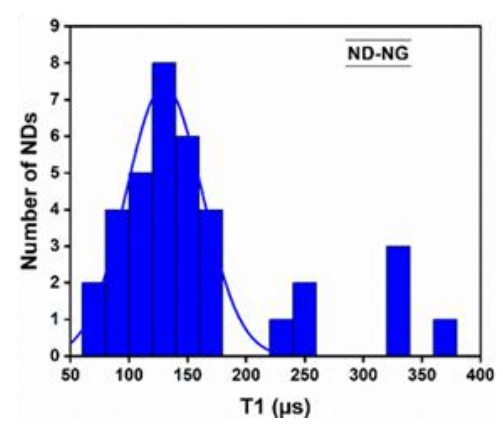

$\mathbf{F}$
B

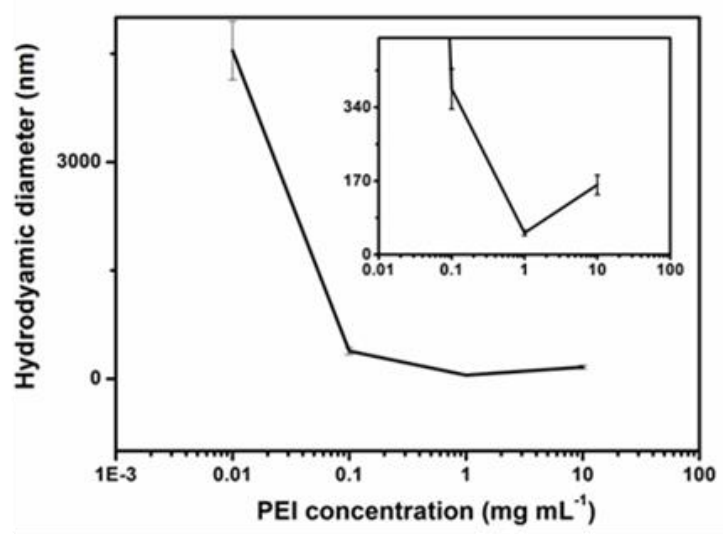

D

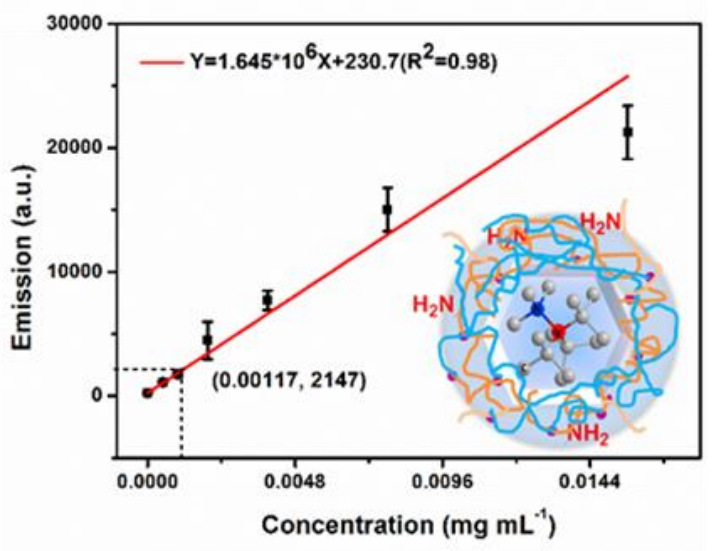

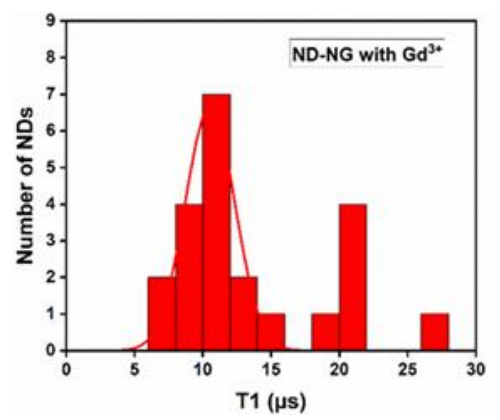

G

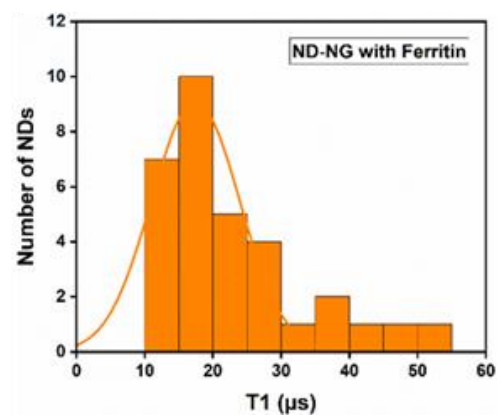

Figure 2. (A) Hydrodynamic diameter of NDs and ND-NGs measured by DLS. (B) Influence of the PEI concentration on the size of ND-NGs. (C) Normalized emission spectra (ex. $532 \mathrm{~nm}$ ) of NDs and ND-NGs. NV ${ }^{-}$zero phonon lines are visible in both spectra. (D) Standard curve of fluorescence intensity using ethylenediamine concentration. (E) $T_{1}$ time of ND-NG (129.47 \pm $32.5 \mu \mathrm{s})$. (F) $T_{1}$ time of ND-NG with $\mathrm{Gd}^{3+}(10.53 \pm 1.82 \mu \mathrm{s})$. (G) $T_{1}$ time of ND-NG with ferritin $(17.43 \pm 6.45 \mu \mathrm{s})$.

Post-functionalization allows controlling the chemical, physical, and physiological properties of ND essential for further applications. ${ }^{[12]}$ PEI possesses many amine groups, which are positively charged at physiological $\mathrm{pH}$. Bare NDs have a zeta potential of $-37.2 \pm 0.6 \mathrm{mV}$, which increased to about $18.8 \pm 0.78 \mathrm{mV}$ (Table S1) after coating, due to many free primary 
amino groups located at the ND-NG surface. Fluorescamine is a commonly used fluorogenic reagent for the detection and quantification of amino groups. An excess of fluorescamine was added to the ND-NGs solution and the fluorescence intensity was measured using an excitation and emission wavelength of $365 \mathrm{~nm}$ and $470 \mathrm{~nm}$ (Figure S6), respectively. In comparison to the control samples, ND-NG, fluorescamine, and water, only the ND-NGs incubated with fluorescamine showed significant fluorescence proving the accessibility of amino groups present on the surface of ND-NGs. Using ethylenediamine to achieve a calibration plot (Figure 2D), the content of the amine groups on ND-NGs was calculated suggesting about $3.90 \times 10^{-5}$ mol primary amino groups $\left(-\mathrm{NH}_{2}\right)$ per gram ND-NGs. Assuming that the NDs consist only of $\mathrm{C}$ atoms and that all ND-NGs particles have a spherical shape of about $40 \mathrm{~nm}$ diameter, ${ }^{[17]}$ this results in an estimated number of about 2,769 amino groups per ND-NG that could be used for post modifications.

The effect of the nanogel shell on the photophysical properties of NDs containing NV centers was investigated (Figure 2C, Figure S5). For optical measurements, ND-NGs with a diameter of $57.0 \pm 1.2 \mathrm{~nm}$ were used. The fluorescence intensity of ND-NGs in water was measured using a laser beam $\left(\lambda_{\mathrm{ex}}=560 \mathrm{~nm}\right.$ and $\lambda_{\mathrm{em}}=680 \mathrm{~nm}$, Figure S5). No significant reduction in the fluorescence intensity was observed for ND-NGs compared to bare NDs. Additionally, no change in the spectral shape was detected. Furthermore, the effect of the nanogel shell on the charge state of NV centers in NDs was studied at the single nanoparticle level (Figure 2C). The spectra were measured on a custom-built confocal microscope with an excitation laser at 532 $\mathrm{nm}$ and $100 \mu \mathrm{W}$ power in front of the objective (oil, NA=1.35). Obviously, the nanogel shell did not affect the emission properties and the zero phonon lines of $\mathrm{NV}^{-}$centers were still well visible without any shift or background noise. NV centers in NDs are very sensitive to the surface states and at some conditions, they can switch to the dark state (positively charged NV center; $\mathrm{NV}^{+}$). These results demonstrate that the nanogel coating did not affect the charge properties of the NV centers, which remained in the optically active states $\left(\mathrm{NV}^{-}\right)$and which is 
important for the future application in bioimaging and nanoscaleD sensing. To prove the nanoscale sensing ability of $\mathrm{ND}-\mathrm{NG}, \mathrm{Gd}^{3+}$ salt and the iron storage protein ferritin containing iron in ferric state were selected exemplarily as paramagnetic species. ND-NG was incubated with $\mathrm{GdCl}_{3}$ or ferritin overnight and purified by centrifugation. While the adsorption of ferritin was revealed by TEM (Figure S8A), $\mathrm{Gd}^{3+}$ ions probably penetrated into the nanogel and were not visible in TEM (Figure S8B+C). To evaluate the influence of the $\mathrm{Gd}^{3+}$ and ferritin adsorbed to ND-NG on the NV spin longitudinal $T_{1}$ relaxation time, we used a confocal microscope equipped to perform $T_{1}$ spin relaxometry. A pulsed $T_{1}$ sequence was chosen, consisting of repetitive laser pulses in absence of a microwave. The spin relaxation from the $m_{\mathrm{s}}=0$ spin state to the thermally mixed state was probed and the relaxometry measurements were performed on randomly selected single particles. For each sample, the resulting $T_{1}$ constants were averaged. The $T_{1}$ time decreased from $129.47 \pm 32.5 \mu$ s of ND-NG to $10.53 \pm 1.82 \mu$ s of ND-NG with complexed $\mathrm{Gd}^{3+}$ and $17.43 \pm 6.45 \mu$ s of ND-NG with ferritin indicating that the ND-NGs are in principle able to sense magnetic fields in their direct surrounding (Figure 2E-G).

Next, the biocompatibility of ND-NGs was investigated using A549 human lung adenocarcinoma cell line (Figure 3). As depicted in Figure 3B and 3C, ND-NGs were efficiently taken up into A549 cells after 4 hours of incubation. We found many homogenously distributed spherical structures indicating that ND-NGs were located in intracellular vesicles. The uptake was dependent on the ND-NG concentration and incubation time. When we increased the incubation time from $6 \mathrm{~h}$ to $24 \mathrm{~h}$ and the concentration of ND-NGs from $100 \mu \mathrm{g}$ $\mathrm{mL}^{-1}$ to $200 \mu \mathrm{g} \mathrm{mL}^{-1}$, the uptake became even more prominent. In addition, cells were proliferating well and the cell morphology was not altered. Low cytotoxicity of ND-NGs was observed after the treatment of the cells with a concentration up to $800 \mu \mathrm{g} \mathrm{mL}^{-1}$ (Figure 3A). To further evaluate the biocompatibility of ND-NG, the Hen's Egg Test on the Chorioallantoic Membrane (HET-CAM) method ${ }^{[29]}$ was chosen, a potential alternative of animal experiments. 
The wide availability of fertile eggs and easily achievable hatching temperature $\left(37-38{ }^{\circ} \mathrm{C}\right)$ made the HET-CAM a desirable experiment platform. The chorioallantoic membrane (CAM) has an ample vascular network which is suitable for studying tissue xenograft, tumor growth, drug delivery, wound healing, and toxicologic study. ${ }^{[30]}$ The CAM is not innervated, and the chick embryo develops a functional brain only on the day 13 of incubation ${ }^{[31]}$. Therefore HETCAM model can be considered as an animal friendly and humane alternative of in vivo testing. In our HET-CAM test, we observed hemorrhage from blood vassals started within 2 - 4 seconds after applying the positive control (1\% sodium dodecyl sulfate; SDS). Lysis occurred after 25 - 27 seconds, and we observed coagulation within 24 hours. For the negative control (phosphate buffered saline) and various concentrations of NG-NG $\left(100 \mu \mathrm{g} \mathrm{mL}^{-1}, 400 \mu \mathrm{g} \mathrm{mL}^{-1}, 800 \mu \mathrm{g} \mathrm{mL}-\right.$ ${ }^{1}$ ) no instance of irritation was detected within 5 minutes to 24 hours of application (Figure 3D and Figure S7). A summary of the results is shown in Table S3. These in ovo results support the biocompatibility of ND-NG. 

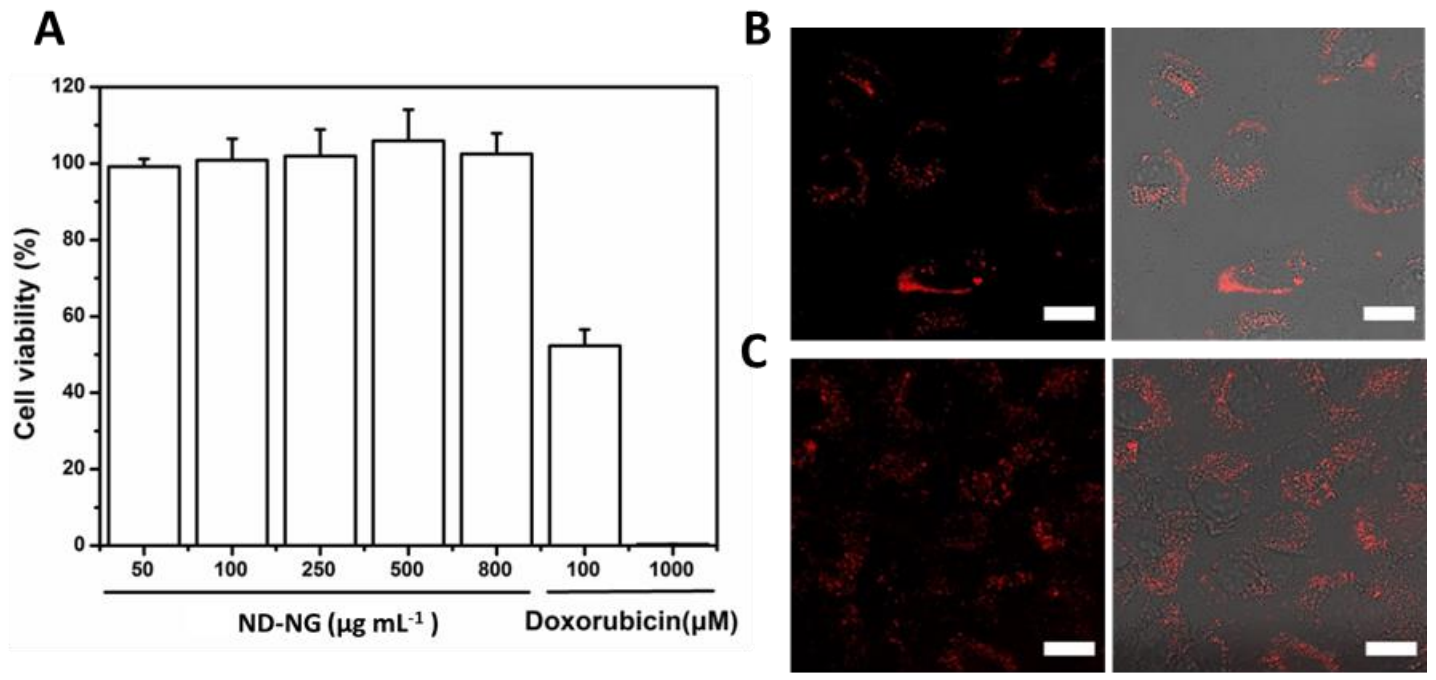

D

$1 \%$ SDS
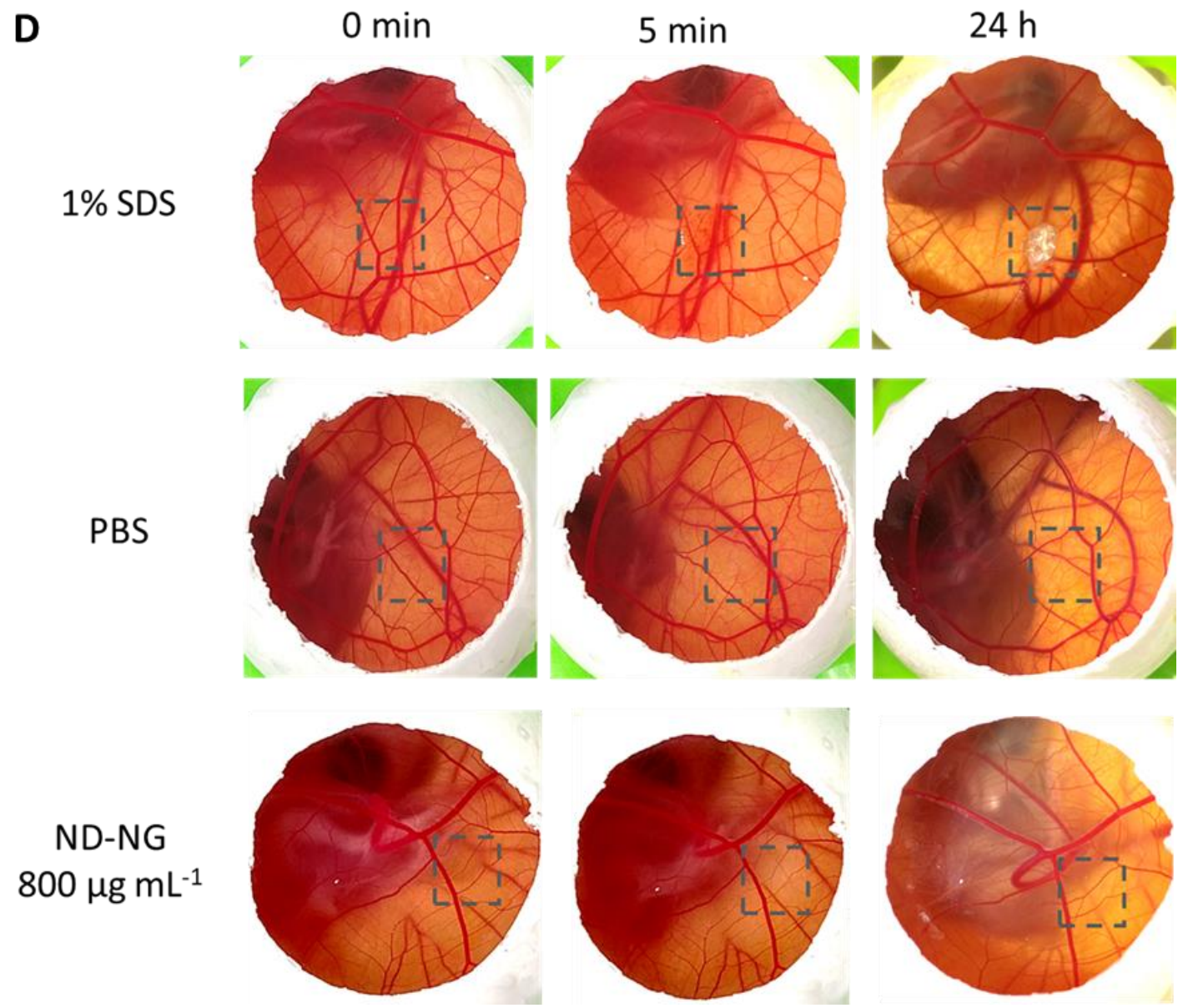

Figure 3. (A) Cell viability of ND-NGs in A549 cells. (B, C) Confocal microscopy images of ND-NGs taken up into A549 cells (scale bar $=20 \mu \mathrm{m}$ ). (B) at $100 \mu \mathrm{g} \mathrm{mL}{ }^{-1}$ for $6 \mathrm{~h}$, (C) at 200 $\mu \mathrm{g} \mathrm{mL} \mathrm{m}^{-1}$ for $24 \mathrm{~h}$. (D) Photographs of HET-CAM test results for $1 \%$ SDS (positive control), PBS (negative control) and ND-NG at $800 \mu \mathrm{g} \mathrm{mL} \mathrm{m}^{-1}$. 
A

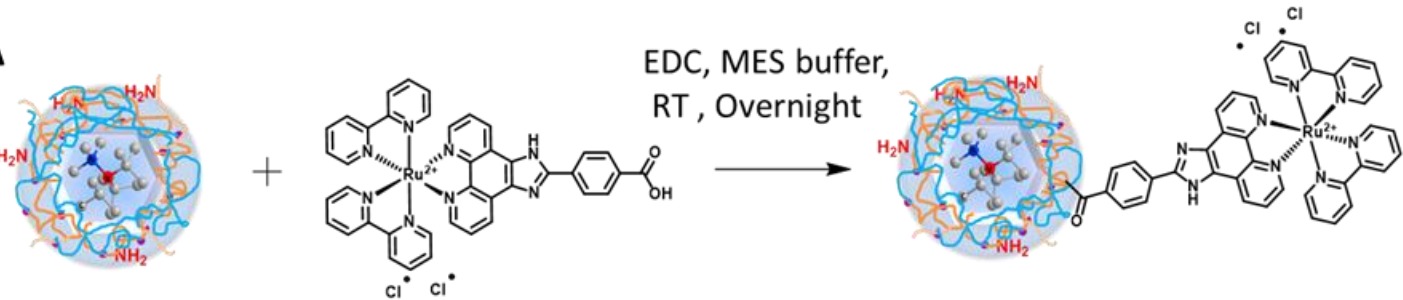

ND-NG

B

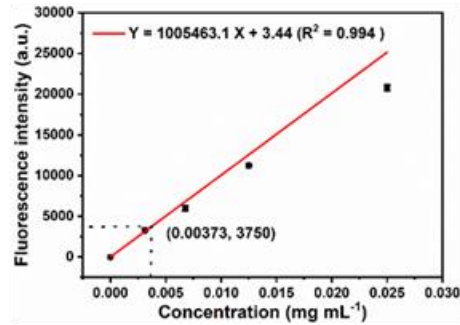

E

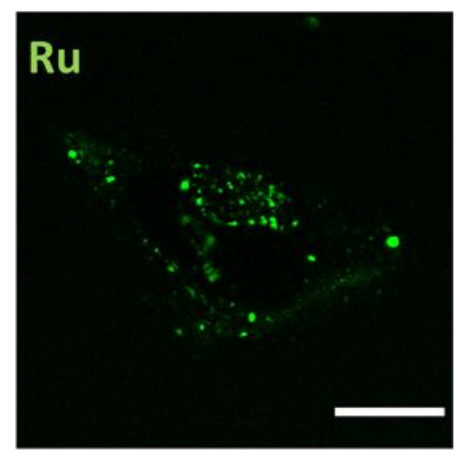

$\mathbf{F}$

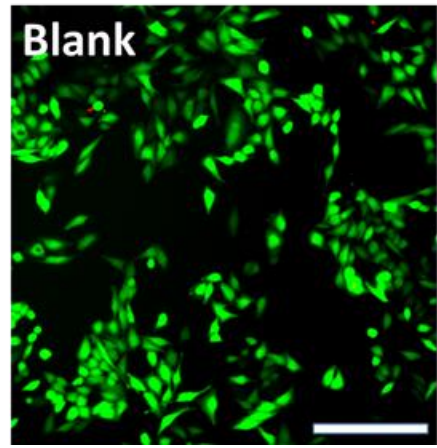

c
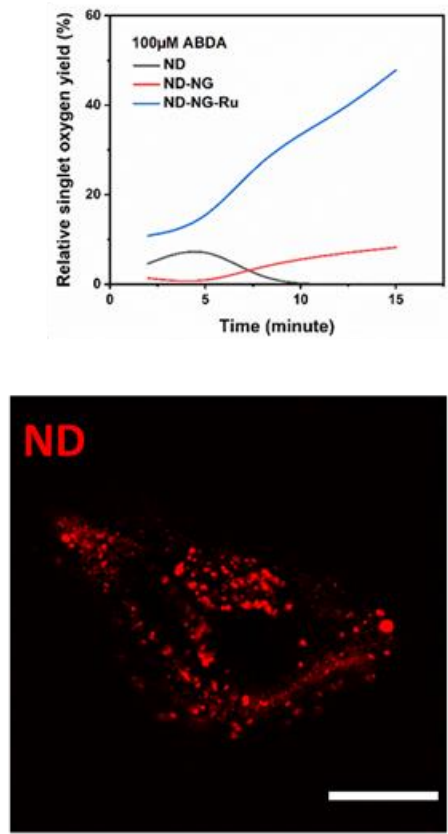

G

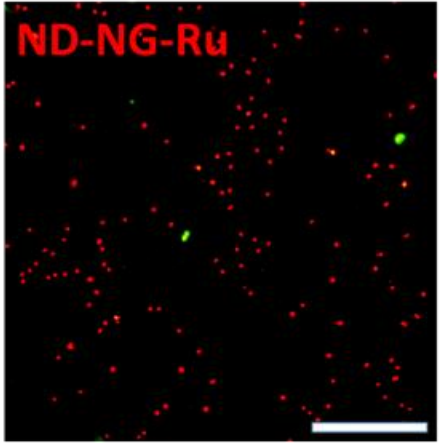

D
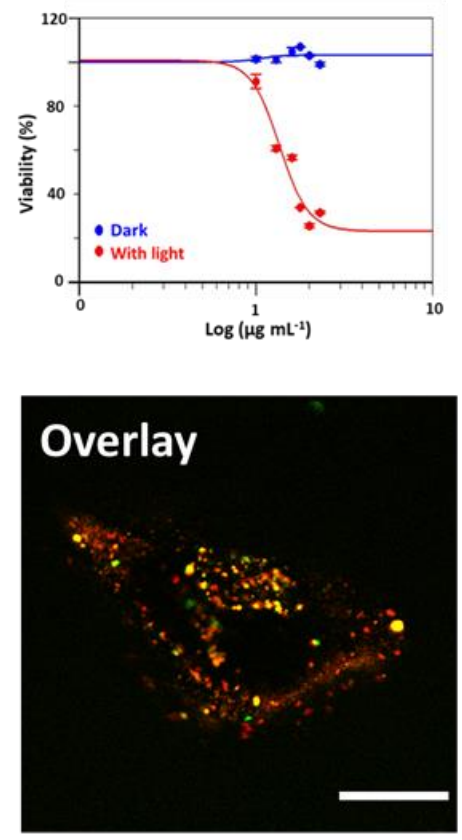

ND-NG-Ru

N

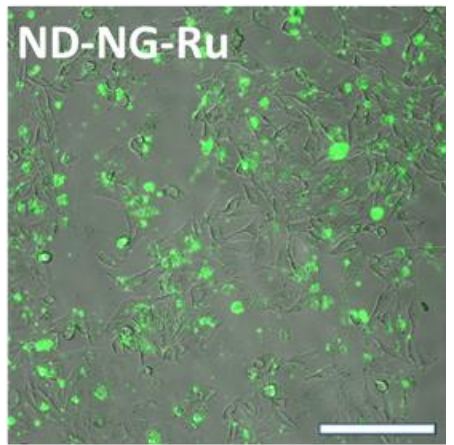

Figure 4. (A) Schematic illustration of the preparation of ND-NG-Ru. (B) Standard curve of fluorescence intensity using different concentration of Ru-COOH. (C) Singlet oxygen yield of $\mathrm{ND}-\mathrm{NG}-\mathrm{Ru}$. (D) Logarithmic fitting curve for cell viability of ND-NG-Ru. (E) Confocal microscopy images in HeLa cells after $4 \mathrm{~h}$ incubation with $100 \mu \mathrm{g} \mathrm{mL}^{-1} \mathrm{ND}-\mathrm{NG}-\mathrm{Ru}(15 \mathrm{~min}$ of irradiation, scale bar $=20 \mu \mathrm{m})$. Green and red colors represent $\mathrm{Ru}$ and ND signal respectively. (F) Live/dead staining of HeLa cells incubated without or with $100 \mu \mathrm{g} \mathrm{mL} \mathrm{m}^{-1} \mathrm{ND}-\mathrm{NG}-\mathrm{Ru}$ after 15 min of irradiation $($ scale bar $=200 \mu \mathrm{m})$. $(\mathrm{G})$ Early apoptosis detection by Annexin V - FITC, Green color represents apoptotic cells (scale bar $=200 \mu \mathrm{m})$.

To underline the potential of ND-NGs as a theranostic platform, a photodynamic therapy (PDT)

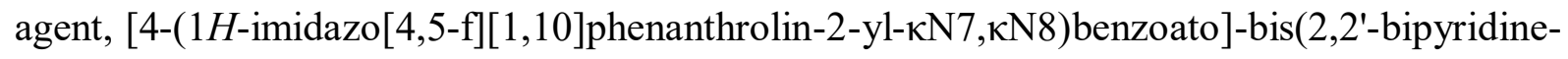


$\kappa \mathrm{N} 1, \kappa \mathrm{N} 1$ ')ruthenium $(1+)$ chloride $(\mathrm{Ru}-\mathrm{COOH})$ was conjugated to the ND-NGs by the reaction of its carboxylic acid with the amine groups of ND-NGs in the presence of EDC to afford NDNG-Ru (Figure 4A). PDT is a treatment using photosensitizing agents, whose bioactivity is activated by light. ${ }^{[32]}$ The photosensitizer produces reactive oxygen species i.e. singlet oxygen $\left({ }^{1} \mathrm{O}_{2}\right)$ for therapeutic purposes. Singlet oxygen is cytotoxic, which strongly decreases i.e. viability of tumor cells. ${ }^{[33]}$ PDT is minimally invasive compared to other treatment procedures (e.g., surgery), and can be used together with other therapeutic techniques. Due to the low invasiveness of this approach, small or no scarring at the application site after healing represents an additional benefit for the patient's quality of life. However, there are some drawbacks including low tumor specificity, inefficient cellular uptake, and higher activation energies (if required) leading to prolonged illumination times. To overcome these challenges, ND-NG was used as a carrier system. After modification with $\mathrm{Ru}-\mathrm{COOH}$, the attenuated total reflection Fourier transform infrared (ATR-FTIR) spectrum of ND-NG-Ru was measured (Figure S9) and showed that the peak intensity of the stretching vibration of $\mathrm{N}-\mathrm{H}$ decreased as the amine group reacted with the carboxylic acid groups of $\mathrm{Ru}-\mathrm{COOH}$. To quantify the number of $\mathrm{Ru}$ complexes on ND-NG-Ru, the fluorescence intensity was measured using an excitation and emission wavelength of $460 \mathrm{~nm}$ and $610 \mathrm{~nm}$, respectively. In comparison to the control samples (NDNG), only ND-NG-Ru showed emission properties in the characteristic range (Figure S10). Using a series of Ru-COOH solutions, a calibration plot was generated (Figure 4B) and the content of the Ru complexes on the ND-NG-Ru was calculated to be $3.78 \mu \mathrm{g} \mathrm{mg}^{-1}$ of ND-NGs. The DLS and TEM measurements of ND-NG-Ru revealed a high colloidal stability with good dispersion and stability in aqueous media (Figure S11 and S12). Compared to the hydrodynamic size of ND-NG, the dimension of ND-NG-Ru increased from $57.0 \pm 1.2 \mathrm{~nm}$ to $73.7 \pm 4.9 \mathrm{~nm}$, respectively. Besides, no significant aggregation was observed in TEM images. PDT relies on the efficient production of singlet oxygen in cellular environments. To monitor the generation of ${ }^{1} \mathrm{O}_{2}$ in a quantitative fashion, we performed ${ }^{1} \mathrm{O}_{2}$ production efficiency tests as 
reported previously. ${ }^{[34]}$ The singlet oxygen sensor 9,10-anthracenediyl-bi(methylene)dimalonic acid (ABDA) was used, which forms an endoperoxide of ABDA in the presence of ${ }^{1} \mathrm{O}_{2}$ that alters its absorbance spectrum. Therefore, the production of ${ }^{1} \mathrm{O}_{2}$ was monitored by measuring this particular change in absorbance of ABDA (Figure 4C). ND-NG-Ru, ND-NGs, and bare NDs as control were mixed with $100 \mu \mathrm{M}$ of ABDA and were irradiated with a $470 \mathrm{~nm}$ LED array $\left(20 \mathrm{~mW} \mathrm{~cm}{ }^{-2}\right)$ for $15 \mathrm{~min}$. Relative changes in absorbance confirmed the successful generation of ${ }^{1} \mathrm{O}_{2}$. We also examined the intracellular localization of ND-NG-Ru in a human cervical cancer cell line (HeLa cells) by laser scanning confocal microscopy. These cells were incubated with ND-NG-Ru $\left(100 \mu \mathrm{g} \mathrm{mL}^{-1}\right)$ for about $4 \mathrm{~h}$ before images were recorded (Figure 4E). ND-NG-Ru were efficiently taken up and many homogenously distributed spherical structures were observed suggestion that ND-NGs were mainly located in intracellular vesicles. To evaluate light induced cellular toxicity, HeLa cells were incubated with 0 - $200 \mu \mathrm{g} \mathrm{mL}^{-1} \mathrm{ND}$ NG-Ru for $4 \mathrm{~h}$ before irradiation with $470 \mathrm{~nm}$ LED light for $15 \mathrm{~min}\left(50 \mathrm{~mW} \mathrm{~cm}{ }^{-2}\right)$. The applied power is comparable to reported photosensitizing drugs. ${ }^{[35]}$ We found a very low $\mathrm{IC}_{50}$ of around $23 \mu \mathrm{g} \mathrm{mL}^{-1}$ for the ND-NG-Ru (Figure 4D), which is similar to the reported $\mathrm{IC}_{50}$ of $15 \mu \mathrm{g} \mathrm{mL}-$ ${ }^{1}$ of the Ru-tetrazole coordinated nanoparticles. ${ }^{[36]}$ Live/dead staining of the cells using fluorescein diacetate/propidium iodide further demonstrated that ND-NG-Ru induced cell death upon irradiation could be spatially controlled (Figure 4F). Furthermore, we confirmed induced cell death using Annexin- $\mathrm{V}$, a protein that binds to phosphatidylserine which is only present during apoptosis (Figure 4G).

\section{Conclusion}

In summary, we have established the adsorption-crosslinking method as a novel synthesis concept for the surface modification of NDs. This approach combines both non-covalent adsorption and covalent stabilization thus integrating the advantages of both coating strategies within one system while overcoming their respective drawbacks. Using this method, we 
successfully prepared ND-NG samples with a thin and uniform nanogel shell as well as a narrow size distribution while elucidating the chemical tools to control its formation. Critically, the photophysical properties of the NV centers in ND were not affected by surface modification thus facilitating the nanoscale sensing applications. Quantification of the number of amine groups, which are presented on the surface of ND-NGs was achieved. Furthermore, the NDNG were well tolerated in cell experiments and in vivo testing. Introduction of a photodynamic therapy agent $\mathrm{Ru}-\mathrm{COOH}$ was achieved in a post-modification approach. ND-NG-Ru showed successful photodynamic activity in vitro. By demonstrating the capability to combine multiple functions i.e. the nanoscale sensing and photodynamic ability, the versatility of the platform was proven. Importantly, the adsorption-crosslinking method can be expanded to other chemical motifs, as reactive groups are still available within the nanogels for further modification. We believe that our studies pave the way to customized ND-based nanotheranostics for precise diagnosis and therapy at the subcellular level.

\section{Conflicts of interest}

The authors declare no competing financial interest.

\section{Supporting Information}

Supporting Information is available from the author.

\section{Acknowledgements}

The authors thank the Polymer Analytics for multiangle DLS measurements, Dr. David Yuen Wah Ng for the fruitful discussion and suggestions, Dr. Gönül Kizilsavas for her support, and Adriana Sobota for her suggestions regarding the HET-CAM assay. The authors are grateful for the financial support from the European Union's Horizon 2020 Research and Innovation Program under FETOPEN grant agreement no. 858149 (AlternativeToGd) and from the Deutsche Forschungsgemeinschaft (DFG, German Research Foundation) - Project number 316249678 - SFB 1279 (C04). Y. Wu thanks the China Scholarship Council for a fellowship.

\section{Author Contributions}

† Yingke Wu and Md Noor A Alam contributed equally. 


\section{References}

[1] a) M. Ibrahim, Y. Xue, M. Ostermann, A. Sauter, D. Steinmueller-Nethl, S. Schweeberg, A. Krueger, M. R. Cimpan, K. Mustafa, J. Biomed. Mater. Res., Part A 2018, 106, 1697; b) K. Adach, M. Fijalkowski, G. Gajek, J. Skolimowski, R. Kontek, A. Blaszczyk, Chem.-Biol. Interact. 2016, 254, 156; c) V. Vaijayanthimala, P.-Y. Cheng, S.-H. Yeh, K.-K. Liu, C.-H. Hsiao, J.-I. Chao, H.-C. Chang, Biomaterials 2012, 33, 7794.

[2] G. Balasubramanian, I. Chan, R. Kolesov, M. Al-Hmoud, J. Tisler, C. Shin, C. Kim, A. Wojcik, P. R. Hemmer, A. Krueger, Nature 2008, 455, 648.

[3] a) S. Han, M. Raabe, L. Hodgson, J. Mantell, P. Verkade, T. Lasser, K. Landfester, T. Weil, I. Lieberwirth, Nano Lett. 2019, 19, 2178; b) M. D. Torelli, N. A. Nunn, O. A. Shenderova, Small 2019, 15, 1902151; c) H. S. Jung, K. J. Cho, Y. Seol, Y. Takagi, A. Dittmore, P. A. Roche, K. C. Neuman, Adv. Funct. Mater. 2018, 28, 1801252.

[4] Y. Wu, A. Ermakova, W. Liu, G. Pramanik, T. M. Vu, A. Kurz, L. McGuinness, B. Naydenov, S. Hafner, R. Reuter, Adv. Funct. Mater. 2015, 25, 6576.

[5] S. Harvey, M. Raabe, A. Ermakova, Y. Wu, T. Zapata, C. Chen, H. Lu, F. Jelezko, D. Y. Ng, T. Weil, Adv. Ther. 2019, 2, 1900067.

[6] a) Y. Zhang, Z. Cui, H. Kong, K. Xia, L. Pan, J. Li, Y. Sun, J. Shi, L. Wang, Y. Zhu, Adv. Mater. 2016, 28, 2699; b) Z. Cui, Y. Zhang, K. Xia, Q. Yan, H. Kong, J. Zhang, X. Zuo, J. Shi, L. Wang, Y. Zhu, Nat. Commun. 2018, 9, 1; c) T. K. Ryu, S. W. Baek, R. H. Kang, S. W. Choi, Adv. Funct. Mater. 2016, 26, 6428.

[7] M. H. Alkahtani, F. Alghannam, L. Jiang, A. Almethen, A. A. Rampersaud, R. Brick, C. L. Gomes, M. O. Scully, P. R. Hemmer, Nanophotonics 2018, 7, 1423.

[8] a) P. Neumann, I. Jakobi, F. Dolde, C. Burk, R. Reuter, G. Waldherr, J. Honert, T. Wolf, A. Brunner, J. H. Shim, Nano Lett. 2013, 13, 2738; b) D. A. Simpson, E. Morrisroe, J. M. McCoey, A. H. Lombard, D. C. Mendis, F. Treussart, L. T. Hall, S. Petrou, L. C. Hollenberg, ACS Nano 2017, 11, 12077; c) T. Sekiguchi, S. Sotoma, Y. Harada, Biophys. Physicobiol. 2018, $15,229$.

[9] a) A. Ermakova, G. Pramanik, J.-M. Cai, G. Algara-Siller, U. Kaiser, T. Weil, Y.-K. Tzeng, H.-C. Chang, L. McGuinness, M. B. Plenio, Nano Lett. 2013, 13, 3305; b) T. Zhang, G.Q. Liu, W.-H. Leong, C.-F. Liu, M.-H. Kwok, T. Ngai, R.-B. Liu, Q. Li, Nat. Commun. 2018, 9, 1 .

[10] a) V. R. Horowitz, B. J. Alemán, D. J. Christle, A. N. Cleland, D. D. Awschalom, Proc. Natl. Acad. Sci. U. S. A. 2012, 109, 13493; b) R. D. Akiel, X. Zhang, C. Abeywardana, V. Stepanov, P. Z. Qin, S. Takahashi, J. Phys. Chem. B 2016, 120, 4003.

[11] K. Xia, C.-F. Liu, W.-H. Leong, M.-H. Kwok, Z.-Y. Yang, X. Feng, R.-B. Liu, Q. Li, Nat. Commun. 2019, 10, 1.

[12] K. van der Laan, M. Hasani, T. Zheng, R. Schirhagl, Small 2018, 14, 1703838.

[13] J. J. Virgen-Ortíz, J. C. dos Santos, Á. Berenguer-Murcia, O. Barbosa, R. C. Rodrigues, R. Fernandez-Lafuente, J. Mater. Chem. B 2017, 5, 7461.

[14] L. Zhao, Y.-H. Xu, T. Akasaka, S. Abe, N. Komatsu, F. Watari, X. Chen, Biomaterials 2014, 35, 5393.

[15] L.-W. Tsai, Y.-C. Lin, E. Perevedentseva, A. Lugovtsov, A. Priezzhev, C.-L. Cheng, Int. J. Mol. Sci. 2016, 17, 1111.

[16] A. Bumb, S. K. Sarkar, N. Billington, M. W. Brechbiel, K. C. Neuman, J. Am. Chem. Soc. 2013, 135, 7815.

[17] L. Zhao, T. Takimoto, M. Ito, N. Kitagawa, T. Kimura, N. Komatsu, Angew. Chem. Int. Ed. 2011, 50, 1388.

[18] X. Q. Zhang, R. Lam, X. Xu, E. K. Chow, H. J. Kim, D. Ho, Adv. Mater. 2011, 23, 4770.

[19] X.-Q. Zhang, M. Chen, R. Lam, X. Xu, E. Osawa, D. Ho, ACS Nano 2009, 3, 2609.

[20] R. A. Shimkunas, E. Robinson, R. Lam, S. Lu, X. Xu, X.-Q. Zhang, H. Huang, E. Osawa, 
D. Ho, Biomaterials 2009, 30, 5720.

[21] J. Ackermann, A. Krueger, Carbon 2020, https://doi.org/10.1016/j.carbon.2020.02.088.

[22] S. R. Hemelaar, A. Nagl, F. Bigot, M. M. Rodríguez-García, M. P. de Vries, M. Chipaux, R. Schirhagl, Microchim. Acta 2017, 184, 1001.

[23] a) P. A. Longo, J. M. Kavran, M.-S. Kim, D. J. Leahy, in Methods in enzymology, Vol. 529, Elsevier, 2013, 227; b) H. Wang, Q. Li, J. Yang, J. Guo, X. Ren, Y. Feng, W. Zhang, J. Mater. Chem. B 2017, 5, 1408.

[24] a) Y. H. Kim, J. H. Park, M. Lee, Y.-H. Kim, T. G. Park, S. W. Kim, Journal of controlled release 2005, 103, 209; b) L. Kong, J. Qiu, W. Sun, J. Yang, M. Shen, L. Wang, X. Shi, Biomater. Sci. 2017, 5, 258; c) W. Sun, Y. Wang, M. Cai, L. Lin, X. Chen, Z. Cao, K. Zhu, X. Shuai, Biomater. Sci. 2017, 5, 2468.

[25] a) C. Graf, D. L. Vossen, A. Imhof, A. van Blaaderen, Langmuir : the ACS journal of surfaces and colloids 2003, 19, 6693; K. M. Koczkur, S. Mourdikoudis, L. Polavarapu, S. E. Skrabalak, Dalton Trans. 2015, 44, 17883; b) M. Farahmandjou, S. Honarbakhsh, S. Behrouzinia, Phys. Chem. Res. 2016, 4, 655.

[26] a) R. Mishra, R. Varshney, N. Das, D. Sircar, P. Roy, Eur. Polym. J. 2019, 119, 155; b) E. Doğan, P. Tokcan, M. Diken, B. Yilmaz, B. Kizilduman, P. Sabaz, Adv. Mater. Sci. 2019, 19, 32; c) Y. Guo, Z. Hao, C. Wan, Tribol. Int. 2016, 93, 214.

[27] F. Heinemann, J. Karges, G. Gasser, Acc. Chem. Res. 2017, 50, 2727.

[28] R. Garcia, R. Proksch, Eur. Polym. J. 2013, 49, 1897.

[29] G. Winter, A. B. Koch, J. Löffler, M. Lindén, C. Solbach, A. Abaei, H. Li, G. Glatting, A. J. Beer, V. Rasche, Cancers 2020, 12, 1248.

[30] A. Vargas, M. Zeisser-Labouèbe, N. Lange, R. Gurny, F. Delie, Adv. Drug Delivery Rev. 2007, 59, 1162.

[31] E. Aleksandrowicz, I. Herr, ALTEX-Alternatives to animal experimentation 2015, 32, 143.

[32] M. C. DeRosa, R. J. Crutchley, Coord. Chem. Rev. 2002, 233, 351.

[33] T. J. Dougherty, C. J. Gomer, B. W. Henderson, G. Jori, D. Kessel, M. Korbelik, J. Moan, Q. Peng, JNCI, J. Natl. Cancer Inst. 1998, 90, 889.

[34] T. Wang, N. Zabarska, Y. Wu, M. Lamla, S. Fischer, K. Monczak, D. Y. Ng, S. Rau, T. Weil, Chem. Commun. 2015, 51, 12552.

[35] S. Chakrabortty, B. K. Agrawalla, A. Stumper, N. M. Vegi, S. Fischer, C. Reichardt, M. Kögler, B. Dietzek, M. Feuring-Buske, C. Buske, J. Am. Chem. Soc. 2017, 139, 2512.

[36] B. Wei, M. Y. Guo, Y. M. Lu, P. P. Sun, G. W. Yang, Q. Y. Li, Anorg. Allg. Chem 2018, 644, 6 . 
A straight-forward synthesis concept called "adsorption-crosslinking" is applied for the modification of ND surfaces, which sequentially combines non-covalent adsorption and subsequent covalent cross-linking. As a result, a thin and uniform nanogel coating surrounding the NDs is obtained, which imparts high colloidal stability and is used for ions and metal protein sensing. After post-functionalization, it is used for photodynamic therapy.

nanodiamonds, nanogels, adsorption-crosslinking, nanoscale sensing, photodynamic applications

Yingke $\mathrm{Wu}, \uparrow \mathrm{Md}$ Noor A Alam, $\uparrow$ Priyadharshini Balasubramanian, Pia Winterwerber, Anna Ermakova, Michael Müller, Manfred Wagner, Fedor Jelezko*, Marco Raabe*, and Tanja Weil*

Fluorescent Nanodiamond-Nanogels for Nanoscale Sensing and Photodynamic Applications

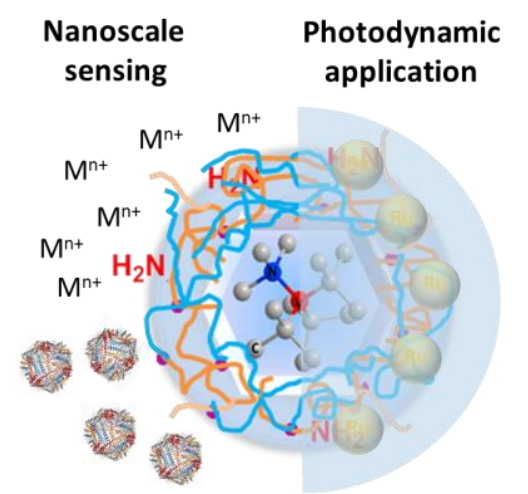

